\title{
Advances in optical imaging for pharmacological studies
}

\begin{abstract}
Alicia Arranz ${ }^{1 *}$ and Jorge Ripoll ${ }^{2,3 *}$
${ }^{1}$ Department of Cell Biology and Immunology, Center for Molecular Biology "Severo Ochoa", Spanish National Research Council, Madrid, Spain, ${ }^{2}$ Department of Bioengineering and Aerospace Engineering, Universidad Carlos III of Madrid, Madrid, Spain, ${ }^{3}$ Experimental Medicine and Surgery Unit, Instituto de Investigación Sanitaria del Hospital Gregorio Marañón, Madrid, Spain
\end{abstract}

Imaging approaches are an essential tool for following up over time representative parameters of in vivo models, providing useful information in pharmacological studies. Main advantages of optical imaging approaches compared to other imaging methods are their safety, straight-forward use and cost-effectiveness. A main drawback, however, is having to deal with the presence of high scattering and high absorption in living tissues. Depending on how these issues are addressed, three different modalities can be differentiated: planar imaging (including fluorescence and bioluminescence in vivo imaging), optical tomography, and optoacoustic approaches. In this review we describe the latest advances in optical in vivo imaging with pharmacological applications, with special focus on the development of new optical imaging probes in order to overcome the strong absorption introduced by different tissue components, especially hemoglobin, and the development of multimodal imaging systems in order to overcome the resolution limitations imposed by scattering.

\footnotetext{
Keywords: bioluminescence, planar fluorescence imaging, fluorescence molecular tomography, optoacoustics, multispectral optoacoustic tomography, multispectral imaging, hybrid systems, data processing
}

\section{Introduction}

Whole-body optical in vivo imaging approaches are valuable tools that enable the study of animal models of human diseases, reducing the number of animals required for experimentation and providing essential information in pharmacological studies. Depending on the physical principle providing image contrast, we find techniques based on light generation, such as bioluminescence or fluorescence imaging, or based on light absorption, such as optoacoustics. All these methodologies enable in vivo imaging of molecular and cellular processes with high sensitivity and have gained great popularity over the past decade mainly because of their safe and straightforward use due to the employment of non-ionizing wavelengths, and their cost-effectiveness compared with other imaging technologies (such as positron emission tomography, PET, or magnetic resonance imaging, MRI; Ntziachristos et al., 2007; Stuker et al., 2011b).

On the other hand, one of the main problems of optical in vivo technologies is dealing with the scattering and absorption properties of tissue (Boas et al., 2011; Ripoll, 2012): scattering is responsible for the loss of light directionality (and therefore a loss in resolution by consequently blurring the image), while the presence of high absorbers (such as melanin and blood) results in a reduction of light intensity (decreasing the signal to noise ratio dramatically in the visible range; Ripoll, 2012).

The most effective way to overcome the loss of signal intensity due to absorption is to employ excitation and emission wavelengths in the near-infrared optical imaging window (between 700 and $900 \mathrm{~nm}$, approximately), where the main tissue constituents (hemoglobin, melanin, water, and lipids) 
absorb the least (Ntziachristos et al., 2005; Jacques, 2013). On the other hand, if one wishes to account for the effects of high scattering in light propagation within tissues in order to obtain a 3D image or quantitative information (note that location, probe concentration, and probe size are strongly interdependent), one needs to introduce a physical model of light propagation within complex media such as a living organism. Once this model is in place, a numerical inversion of this model (what is termed, "solving the inverse problem") is needed in order to obtain a 3D image providing the spatial distribution of probe concentration. Depending on the algorithm we use to reconstruct an image we will be able to recover probe size, position and concentration with varying accuracy. How this issue is addressed clearly distinguishes the different imaging approaches in optical in vivo imaging into the following three categories: (1) planar optical imaging, (2) optical tomography, and (3) optoacoustic tomography.

In this review we discuss the latest advances of optical in vivo imaging as a tool in pharmaceutical studies, addressing the different approaches that are being developed in order to overcome the strong absorption introduced by hemoglobin and the ill-posedness introduced by scattering, either through the use of multimodal imaging or photoacoustic tomography, or by developing new probes or proteins more adequate for in vivo imaging in deep tissues.

\section{Planar Optical Imaging}

Planar optical imaging techniques are by far the most common, mainly due to their simplicity of use and low cost. Two planar imaging modalities are available, depending on the light source generation: Bioluminescence and Fluorescence. In both cases a high sensitivity camera (CCD mainly) coupled to a high numerical aperture camera objective takes a single long exposure image, in the case of fluorescence using appropriate band-pass filters. In what follows we detail recent advances and applications in both modalities.

\section{Bioluminescence In Vivo Imaging}

Bioluminescence imaging is based on the oxidation of a substrate (luciferin) mediated by an enzyme (luciferase), being the most commonly used the luciferase originated from the North American firefly (Photinus pyralis). The firefly luciferase requires ATP and magnesium to catalyze the reaction that leads to the emission of light, which ranges from 530 to $640 \mathrm{~nm}$, depending amongst other factors on the $\mathrm{pH}$, polarity of the solvent, and the microenvironment of the enzyme (Li et al., 2013). Note how this emission falls within the portion of the visible spectrum where hemoglobin is strongly absorbing.

Since the firefly luciferase was cloned (de Wet et al., 1985), the luc gene has extensively been used in gene regulation studies. Bioluminescent probes have also been engineered in order to detect specific enzymatic activities. These probes are designed in such a way that the luciferin is "caged" and this conjugate has to be cleaved by an enzymatic activity (i.e., proteases such as caspases). Once cleaved, the luciferin can be oxidized by the luciferase and the signal is released (Li et al., 2013).
Techniques based on bioluminescence detection have largely been used for molecular biology assays in laboratories worldwide. Accordingly, bioluminescence has also been a reference method for in vivo imaging. Its main advantage is the absence of background signal (the commonly used cell or animal models do not express luciferase and therefore there is no "autobioluminescence"), which leads to a high specificity of the detected signals and an elevated signal-to-noise ratio. This has resulted in an impressive expansion of bioluminescence in vivo imaging applications for studies in cancer biology, inflammation, and infection, amongst others (Edinger et al., 2002; Andreu et al., 2010; Luker and Luker, 2011; Luwor et al., 2015). However, researchers using bioluminescence in vivo imaging have to deal with problems derived from the complexity of the luciferaseluciferin reaction and the effects of light propagation in living tissues. Regarding the luciferase-luciferin reaction, both substrate and co-factors (ATP, oxygen and magnesium) are required for the reaction to take place and therefore the limitation of any of them may result in altered readouts that are not a real representation of luciferase activity (Sadikot and Blackwell, 2005). There have also been significant efforts toward the development of bioluminescence tomography (BLT) approaches, requiring the prior knowledge of one of the parameters or the number of sources in order to produce a 3D image (Liu et al., 2010).

\section{Fluorescence In Vivo Imaging}

After a fluorescent agent is excited with a light source, fluorescence is emitted isotropically as a consequence of a radiative transition from an excited singlet state to a singlet state of lower energy (typically the ground state) following Stoke's Law (Sauer et al., 2011). Even though fluorescence has been extensively used in microscopy for over a century to study molecular and cellular processes (Masters, 2009), it has not been until this past decade that its use for in vivo small animal imaging became significant (Mahmood et al., 1999; Weissleder et al., 1999; Ntziachristos et al., 2005). The high sensitivity offered by this technique and the latest advances in fluorescence labeling have also promoted its relatively recent incursion in non-invasive in vivo imaging. Both planar and three-dimensional fluorescence imaging methods in vivo are now commonly used in pre-clinical research.

In order to acquire a fluorescence image, either as part of a tomographic data set or a single planar image, one requires an excitation source as close as possible to the excitation maximum of the fluorophore being used, if possible within the near infrared optical imaging window. The use of this excitation wavelength, however, will not only excite specifically the fluorophore but will generate non-specific fluorescence from several components present in tissue, generating what is termed "auto-fluorescence," reducing the signal to background ratio (i.e., the contrast in the image). One way to reduce this problem is performing several spectral measurements with different excitation/emission pairs and unmixing the specific signal of the fluorophore from the un-specific signal of the surrounding tissue (Xu and Rice, 2009).

With respect to recent pharmacological studies, Zhang et al. (2015b) make use of planar fluorescence molecular imaging to monitor therapy in murine models of Alzheimer's disease. In particular, the authors verify the feasibility of using CRANAD-3 
A

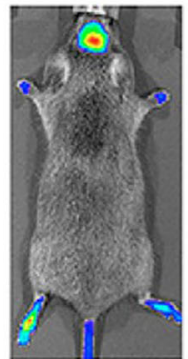

Before

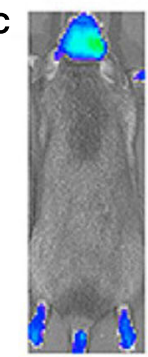

WT

APP

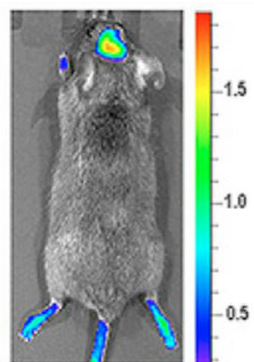

+ LY281376

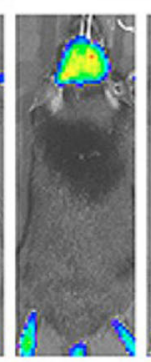

Contrl

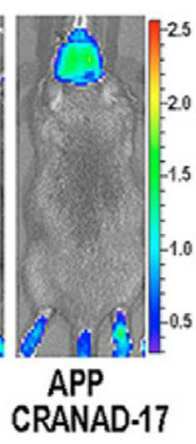

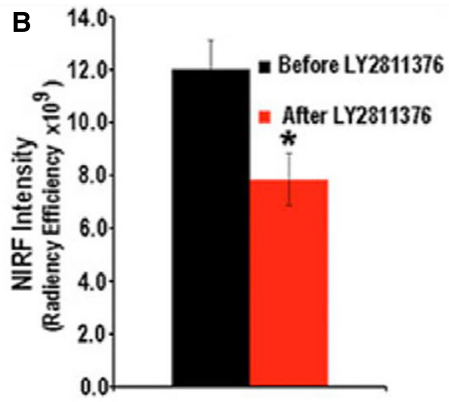

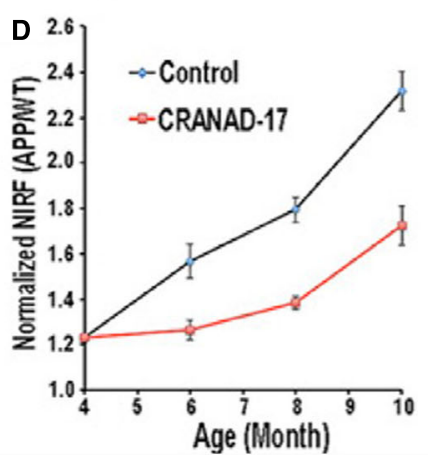

E
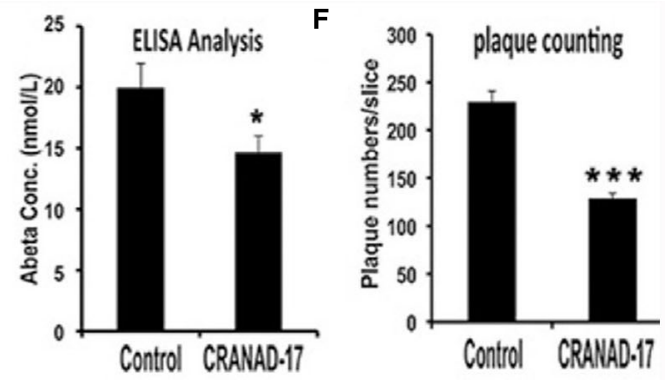

G

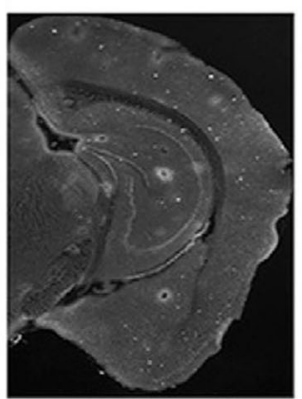

CRANAD-17

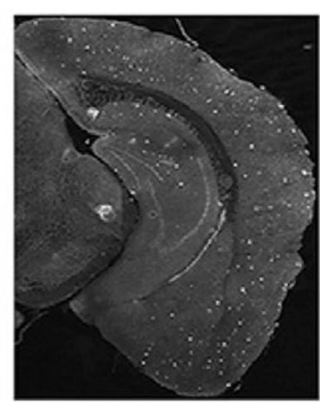

Control

FIGURE 1 | In vivo monitoring of therapeutic effect of drug treatment in Alzheimer's disease. Application of CRANAD-3 for monitoring therapeutic effects of drug treatments. (A) In vivo imaging of APP/PS1 mice with CRANAD-3 before and after treatment with the BACE-1 inhibitor LY2811376. (B) Quantitative analysis of the imaging in A ( $n=4)$. (C) Representative images of 4-month-old APP/PS1 mice after 6 months of treatment with CRANAD-17. (Left) Age-matched WT mouse. (Center) Control APP/PS1 mouse. (Right) CRANAD-17-treated APP/PS1 mouse. Note that the NIRF signal from the CRANAD-17-treated APP/PS1 mouse (Right) is lower than the signal from the non-treated control APP/PS1 mouse (Center). (D) Quantitative analysis of the imaging in C ( $n=5)$. (E) ELISA analysis of total A 340 from brain extracts. (F) Analysis of plaque counting. (G) Representative histological staining with thioflavin S. (Left) CRANAD-17-treated mouse. (Right) Control. ${ }^{\star} P<0.05,{ }^{\star \star} P<0.01,{ }^{\star \star \star} P<0.005$. From Zhang et al. (2015b).

for monitoring therapy, and use it to monitor the therapeutic effect of CRANAD-17, a curcumin analog for inhibition of $\mathrm{A} \beta$ cross-linking (see Figure 1).

\section{Diffuse Optical Tomography and Fluorescence Molecular Tomography}

In order to account for the effect of scattering when imaging tissues with light, diffuse optical tomography (DOT) was developed, based on scanning a point source over the sample and measuring the intensity of the diffuse light either by fibers or with a camera focused onto the surface (see Arridge, 1999, for a review on this subject). With its first applications being targeted toward breast cancer (see, for example Ntziachristos et al., 2000) its use in small animal imaging came with the development of fluorescence molecular tomography, first published in 2002 (Ntziachristos et al., 2002), in the context of molecular imaging by employing an activatable probe to image protease activity in an in vivo mouse model of glioblastoma. Since this first publication in 2002 there have been several developments and applications, mainly in tumor biology (Ntziachristos et al., 2004; Deliolanis et al., 2006; Montet et al., 2007; Kossodo et al., 2010; Hensley et al., 2012) and inflammation studies (Martin et al., 2008; Kang et al., 2014; Thomas et al., 2015), amongst others.

Apart from suffering from auto-fluorescence in a manner similar to planar fluorescence imaging, DOT and FMT provide no anatomical information and therefore benefit from its combination with measurements provided by other imaging systems such as X-ray computed tomography (CT) or MRI, issue which we will discuss at the end of this review. Additionally, the prior knowledge of anatomical features and optical properties significantly improves image quality and quantitation, as will be discussed later.

\section{Optoacoustic In Vivo Imaging}

Being based on the emission of sound after a transient increase in volume due to light absorption, the photoacoustic effect may be used to image in $3 \mathrm{D}$ the location and relative concentration of fluorescence probes using advanced acoustic transducers and light sources. Termed Optoacoustic or Photoacoustic imaging, it circumvents the "blurring" caused by scattering on the visible wavelengths by measuring the acoustic wave generated, which suffers several orders of magnitude less scattering, resulting in an increased penetration depth with no significant loss of signal to noise. In order for this approach to be implemented and transient volume changes generated, we need to use pulsed lasers and then record the ultrasound wave generated by the localized absorption of this pulse of light by the tissue. Recording this ultrasound wave at several locations simultaneously, we may make use of tomographic methods to recover a $3 \mathrm{D}$ image (Wang et al., 2003). When multispectral methods are used, such 

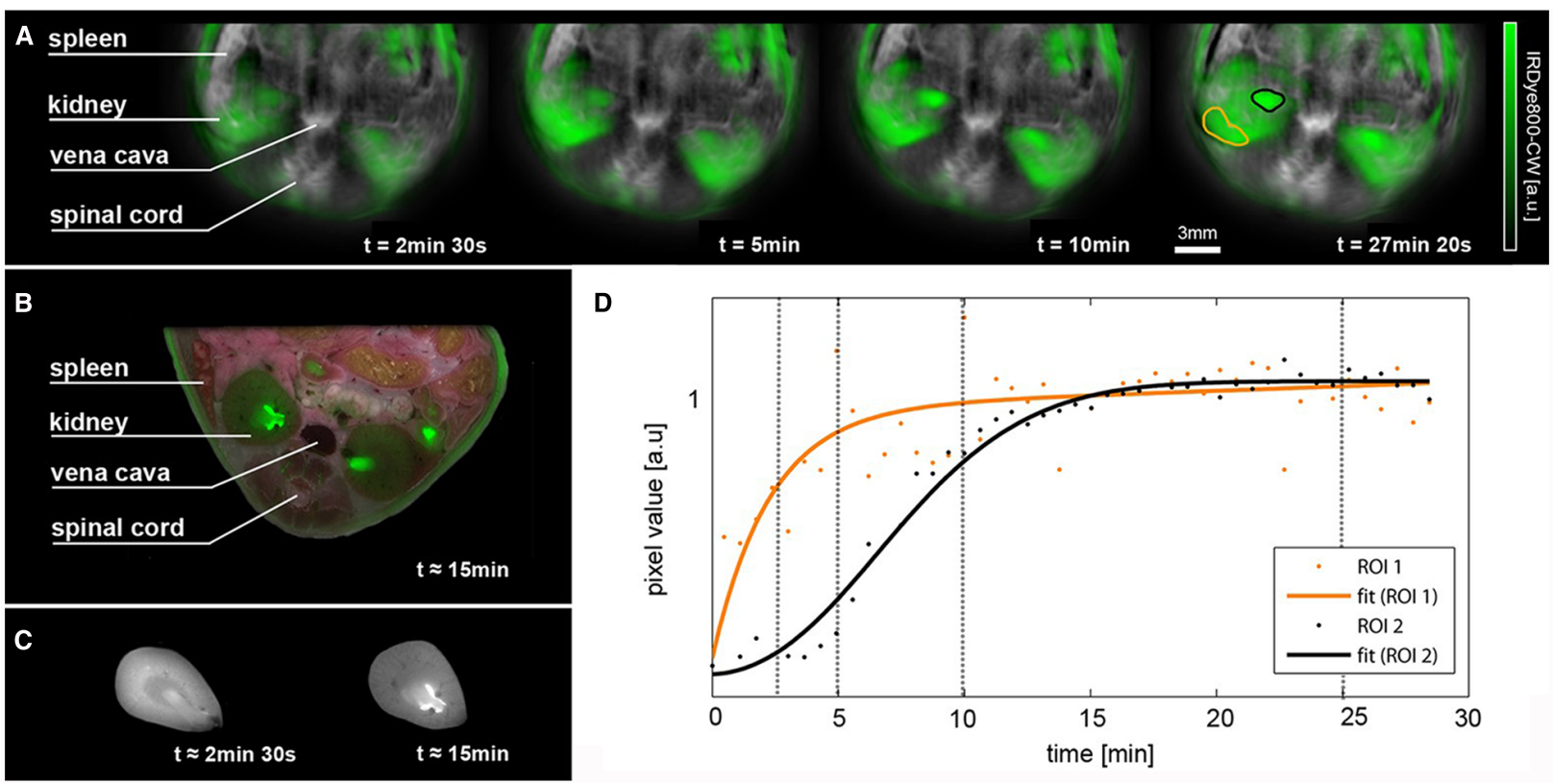

FIGURE 2 | Pharmacokinetic in vivo imaging using MSOT. (A) Time series of images visualizing the biodistribution of IRdye800 in green on logarithmic scale overlaid on the vasculature. Both channels are the result of spectral unmixing. (B) Cryoslice image after approximately 15 min with overlaid fluorescence as a verification of the MSOT results. (C) A comparison of fluorescence distribution in the kidneys of mice sacrificed after approximately 2 min $30 \mathrm{~s}$ after injection and 15 min after injection. Note the changes in distribution similar to the time series shown in (A). (D) Temporal evolution of signal (each normalized to their smoothed maxima) in the regions of interest highlighted in the rightmost image, orange showing a region in the renal cortex that displays early and steep signal pickup and black indicating a region in the renal pelvis where probe accumulation is delayed and has a smoother profile. Time points of the images in (A) are marked using vertical lines. From Taruttis et al. (2012)

as in multispectral optoacoustic tomography (MSOT), different fluorophores may be separated and their relative concentration quantified (Laufer et al., 2007; Ma et al., 2009; Tzoumas et al., 2014), underlying the use of MSOT for quantitative and highly specific in vivo imaging. Additionally, since hemoglobin is a strong absorber, optoacoustic tomography may also be used for resolving vascular structures and quantifying oxygen saturation and blood volume (Lao et al., 2008; Hu and Wang, 2010). The high resolution of MSOT-approximately $\sim 100 \mu \mathrm{m}$ and in some cases even better $[\sim 40 \mu \mathrm{m}$ resolution was shown in $\mathrm{Ma}$ et al. (2009)], good anatomical information, and quantitative 3D images are the reason why this approach is becoming widespread in pharmacological studies.

One application of MSOT to pharmacological studies which show extremely high impact is the use of MSOT to follow pharmacokinetics in vivo (Kossodo et al., 2010; Razansky et al., 2011, 2012; Bednar and Ntziachristos, 2012; Taruttis et al., 2012). Figure 2 shows an example of the potential of MSOT, where a time series of images visualizing in vivo the biodistribution of IRdye 800 and vasculature are shown. This study shows how the spatially localized temporal evolution of drug delivery may be imaged in real time.

One of the drawbacks of optoacoustic tomography is its lower sensitivity when compared to pure fluorescence measurements and the difficulty of imaging in organs that present high acoustic contrast or high impedance mismatch, such as the lungs. Another drawback is that the signal generated is proportional to the light intensity that has been absorbed locally and thus decreases for deeper tissues. Even though the lack of knowledge on the precise light distribution within the subject precludes this technique from being fully quantitative, the development of advanced inverse methods and imaging approaches are constantly improving the quantitative nature of MSOT (Razansky et al., 2011).

\section{Latest Advances to Improve Quantification and Resolution}

Once we have covered the main optical imaging approaches, we now will present the most recent advances to improve these imaging techniques either by changing the emission spectra of the probes or by including anatomical information and thus reducing the ill-posed nature of the inverse problem.

\section{Avoiding Absorption in Living Tissues: Moving Toward the Near Infra-Red}

As mentioned previously, working with wavelengths in the near infra-red (NIR), in particular in the 700-900 $\mathrm{nm}$ window, reduces the amount of light absorbed in tissues by $\sim 3$ orders of magnitude when compared to the visible spectrum. Due mainly to hemoglobin absorption and considering that the emission peak of the native firefly luciferase is in the range of $\sim 562 \mathrm{~nm}$, its detection is mainly limited to the surface. Great efforts have been focused on obtaining mutated versions of luciferase enzymes leading to red-shifted emission wavelengths, with emission peaks above $600 \mathrm{~nm}$ (Branchini et al., 2010a; Stepanyuk et al., 2010; 
Mezzanotte et al., 2011; Wang et al., 2013). In order to obtain emitted light with longer-wavelengths, considerable effort has also been devoted to the development of analogs of the substrate (luciferin), such as aminoluciferins (Mofford et al., 2014) or selenium analogs (Conley et al., 2012). Other developments have been bioluminescence resonance energy transfer (BRET) conjugates, consisting on using the emitted bioluminescence light as excitation for fluorescent molecules. The use of these conjugates results in a final emitted light above $700 \mathrm{~nm}$ (Branchini et al., 2010b), although it has been discussed that they may alter the cellular uptake properties of the substrate (Conley et al., 2012).

In the case of fluorescence, an impressive development of new NIR fluorescent agents has taken place in recent years with excitation maxima above $650 \mathrm{~nm}$, allowing the use of excitation sources and emission spectra within the optical window of the spectrum, where blood absorption is reduced to a minimum (Ntziachristos et al., 2005; Jacques, 2013). Researchers can now benefit from a wide portfolio of near infra-red fluorescent (NIRF) probes designed to be non-targeted (non-specific used for imaging of perfusion or vascular leakage), targeted (such as fluorescent-conjugated antibodies, which recognize and bind specific ligands), or activatable (the fluorescent signal is quenched unless a specific enzymatic activity cleaves the probe). Moreover, different approaches have been followed to obtain NIRF proteins, reaching excitation maxima above $670 \mathrm{~nm}$ (Shcherbo et al., 2007; Shu et al., 2009; Filonov et al., 2012). Constructs for the expression of these proteins and recently developed transgenic mice (Diéguez-Hurtado et al., 2011; Tran et al., 2014) provide an excellent tool for in vivo imaging applied to biomedical and pharmaceutical studies.

With respect to optoacoustics, all advances in fluorescent probes are directly compatible with this methodology, since probes with high quantum yield by definition present high absorption properties. Additionally, optoacoustic imaging methods are also benefiting from new engineered acoustic probes based on metallic nanoparticles (mainly gold) which exhibit high absorption profiles (Bao et al., 2013; Vonnemann et al., 2014).

Finally, a very interesting and promising new development is the use of Cherenkov excited luminescence imaging (CELSI) to improve resolution and partially avoid the effect of absorption while exciting the fluorophores (Zhang et al., 2012, 2013b, 2015a). This approach makes use of Cherenkov emitted NIR light from collimated ionizing radiation generated in a linear accelerator (LINAC), a technique which could potentially be applied for imaging fluorescent markers deep in tissue with high resolution.

\section{Hybrid Systems}

The combination of optical imaging modalities with structural imaging methods such as X-ray CT or MRI allows obtaining anatomical information that can be used as prior data on the reconstruction algorithm to improve both resolution and sensitivity (Ale et al., 2012).

For example, FMT-MRI hybrid systems have been developed and used to analyze protease activity and tumor morphology in mouse tumor models (Davis et al., 2010; Stuker et al., 2011a) or metalloproteinase activity in mouse models of atherosclerosis (Li et al., 2014). FMT-XCT hybrid systems are also examples
TABLE 1 | Comparison of different imaging modalities.

\begin{tabular}{lrlll}
\hline Technique & Resolution & Throughput & Pharmacokinetics & 3D Info \\
\hline Bioluminescence & $>5 \mathrm{~mm}^{*}$ & High & No & No \\
Planar fluorescence & $>5 \mathrm{~mm}^{*}$ & High & No & No \\
FMT & $1-2 \mathrm{~mm}$ & Medium & No & Yes \\
FMT/XCT & $1 \mathrm{~mm}$ & Low & No & Yes \\
MSOT & $0.1 \mathrm{~mm}$ & Low & Yes & Yes \\
\hline
\end{tabular}

${ }^{*}$ Resolution depends on depth location.

where we can make use of anatomical priors obtained from the geometric information provided by the XCT measurements in order to improve the $3 \mathrm{D}$ reconstruction of the fluorescence signal (Ale et al., 2012; Zhang et al., 2013a, 2014).

The technical complexity of these hybrid systems (for example, due to crosstalk between optical and MRI imaging) has led to the use of adapted animal holders which are compatible with different modality systems enabling sequential imaging (McCann et al., 2009).

\section{Conclusions and Future Outlook}

A wide range of optical imaging modalities are available for in vivo imaging in small animals, representing an essential tool in pharmacological studies. Each modality, however, presents its own drawbacks, mainly due to the effects of absorption and scattering of light propagation in living tissues. As shown in Table 1, the selection of a technique will depend on the model used and the information that we want to obtain. For example, if highthroughput imaging is required, planar imaging approaches will be useful, with the consequence that no quantitative information or depth location may be inferred (see Table 1). If quantitative imaging and probe location is important, tomography is needed and FMT and similar approaches are a good option, reaching their full potential when combined with an anatomical imaging modality such as MRI or X-ray CT. As a quickly growing modality, optoacoustic tomography and in particular MSOT shows great potential, so far offering the best imaging resolution, but with the problems associated with ultrasound imaging such as high impedance mismatch in some organs such as the lungs and the need for a matching gels.

We believe that as more specific near infrared fluorescent probes and proteins with distinct spectral features, and specific nanoparticles for high and specific optoacoustic signal generation are generated there will be further improvement of the performance of the technologies covered in this review, opening opportunities for new applications. The combination of several imaging modalities, specifically if they include optical imaging approaches, will ensure the sensitivity and specificity that optical probes uniquely offer may reach their full potential as imaging agents for $3 \mathrm{D}$ quantitative imaging in vivo.

\section{Acknowledgments}

JR acknowledges support from the EC FP7 CIG grant HIGHTHROUGHPUT TOMO, and MINECO grant FIS2013-41802-R MESO-IMAGING. 


\section{References}

Ale, A., Ermolayev, V., Herzog, E., Cohrs, C., de Angelis, M. H., and Ntziachristos, V. (2012). FMT-XCT: in vivo animal studies with hybrid fluorescence molecular tomography-X-ray computed tomography. Nat. Methods 9, 615-620. doi: 10.1038/nmeth.2014

Andreu, N., Zelmer, A., Fletcher, T., Elkington, P. T., Ward, T. H., Ripoll, J., et al. (2010). Optimisation of bioluminescent reporters for use with mycobacteria. PLoS ONE 5:e10777. doi: 10.1371/journal.pone.0010777

Arridge, S. R. (1999). Optical tomography in medical imaging. Inverse Probl. 15, R41-R93. doi: 10.1088/0266-5611/15/2/022

Bao, C., Beziere, N., Del Pino, P., Pelaz, B., Estrada, G., Tian, F., et al. (2013). Gold nanoprisms as optoacoustic signal nanoamplifiers for in vivo bioimaging of gastrointestinal cancers. Small 9, 68-74. doi: 10.1002/smll.201201779

Bednar, B., and Ntziachristos, V. (2012). Opto-acoustic imaging of drug discovery biomarkers. Curr. Pharm. Biotechnol. 13, 2117-2127. doi: $10.2174 / 138920112802502079$

Boas, D. A., Pitris, C., and Ramanujam, N. (eds). (2011). Handbook of Biomedical Optics. Boca Raton: CRC Press.

Branchini, B. R., Ablamsky, D. M., Davis, A. L., Southworth, T. L., Butler, B., Fan, F., et al. (2010a). Red-emitting luciferases for bioluminescence reporter and imaging applications. Anal. Biochem. 396, 290-297. doi: 10.1016/j.ab.2009.09.009

Branchini, B. R., Ablamsky, D. M., and Rosenberg, J. C. (2010b). Chemically modified firefly luciferase is an efficient source of near-infrared light. Bioconjug. Chem. 21, 2023-2030. doi: 10.1021/bc100256d

Conley, N. R., Dragulescu-Andrasi, A., Rao, J., and Moerner, W. E. (2012). A selenium analogue of firefly D-luciferin with red-shifted bioluminescence emission. Angew. Chem. Int. Ed. Engl. 51, 3350-3353. doi: 10.1002/anie.201105653

Davis, S. C., Samkoe, K. S., Hara, J. A. O., Gibbs, S. L., Payne, H. L., Hoopes, P. J., et al. (2010). MRI-coupled fluorescence tomography quantifies EGFR activity in brain tumors. Acad. Radiol. 17, 1-10. doi: 10.1016/j.acra.2009.11.001

Deliolanis, N., Lasser, T., Niedre, M., Soubret, A., and Ntziachristos, V. (2006). In-vivo lung cancer imaging in mice using $360 \hat{\mathrm{A}}^{\circ}$ free-space fluorescence molecular tomography. Conf. Proc. IEEE Eng. Med. Biol. Soc. 1, 2370-2372. doi: 10.1109/IEMBS.2006.260683

de Wet, J. R., Wood, K. V., Helinski, D. R., and DeLuca, M. (1985). Cloning of firefly luciferase cDNA and the expression of active luciferase in Escherichia coli. Proc. Natl. Acad. Sci. U.S.A. 82, 7870-7873. doi: 10.1073/pnas.82.23.7870

Diéguez-Hurtado, R., Martín, J., Martínez-Corral, I., Martínez, M. D., Megías, D., Olmeda, D., et al. (2011). A Cre-reporter transgenic mouse expressing the far-red fluorescent protein Katushka. Genesis 49, 36-45. doi: 10.1002/dvg.20685

Edinger, M., Cao, Y. A., Hornig, Y. S., Jenkins, D. E., Verneris, M. R., Bachmann, M. H., et al. (2002). Advancing animal models of neoplasia through in vivo bioluminescence imaging. Eur. J. Cancer 38, 2128-2136. doi: 10.1016/S09598049(02)00410-0

Filonov, G., Piatkevich, K., Ting, L. M., Zhang, J., Kim, J., and Verkhusha, V. (2012). Bright and stable near infra-red fluorescent protein for in vivo imaging. Nat. Biotechnol. 29, 757-761. doi: 10.1038/nbt.1918

Hensley, H. H., Roder, N. A., Brien, S. W. O., Bickel, L. E., Xiao, F., Litwin, S., et al. (2012). Combined in vivo molecular and anatomic imaging for detection of ovarian carcinoma-associated protease activity and integrin expression in mice. Neoplasia 14, 451-462. doi: 10.1596/neo.12480

$\mathrm{Hu}, \mathrm{S}$., and Wang, L. V. (2010). Photoacoustic imaging and characterization of the microvasculature. J. Biomed. Opt. 15, 1-15. doi: 10.1117/1.3281673

Jacques, S. L. (2013). Optical properties of biological tissues: a review. Phys. Med. Biol. 58, 5007-5008. doi: 10.1088/0031-9155/58/14/5007

Kang, N.-Y., Park, S.-J., Ang, X. W. E., Samanta, A., Driessen, W. H. P., Ntziachristos, V., et al. (2014). A macrophage uptaking near-infrared chemical probe for in vivo imaging of inflammation. Chem. Commun. (Camb.). 50, 6589-6591. doi: $10.1039 / \mathrm{c} 4 \mathrm{cc} 02038 \mathrm{c}$

Kossodo, S., Pickarski, M., Lin, S. A., Gleason, A., Gaspar, R., Buono, C., et al. (2010). Dual in vivo quantification of integrin-targeted and protease-activated agents in cancer using fluorescence molecular tomography (FMT). Mol. Imaging Biol. 12, 488-499. doi: 10.1007/s11307-009-0279-Z

Lao, Y., Xing, D., Yang, S., and Xiang, L. (2008). Noninvasive photoacoustic imaging of the developing vasculature during early tumor growth. Phys. Med. Biol. 53, 4203-4212. doi: 10.1088/0031-9155/53/15/013
Laufer, J., Delpy, D., Elwell, C., and Beard, P. (2007). Quantitative spatially resolved measurement of tissue chromophore concentrations using photoacoustic spectroscopy: application to the measurement of blood oxygenation and haemoglobin concentration. Phys. Med. Biol. 52, 141-168. doi: 10.1088/00319155/52/1/010

Li, B., Maafi, F., Berti, R., Pouliot, P., Rhéaume, E., Tardif, J.-C., et al. (2014). Hybrid FMT-MRI applied to in vivo atherosclerosis imaging. Biomed. Opt. Express 5, 1664. doi: 10.1364/BOE.5.001664

Li, J., Chen, L., Du, L., and Li, M. (2013). Cage the firefly luciferin!-a strategy for developing bioluminescent probes. Chem. Soc. Rev. 42, 662-676. doi: 10.1039/C2CS35249D

Liu, J., Wang, Y., Qu, X., Li, X., Ma, X., Han, R., et al. (2010). In vivo quantitative bioluminescence tomography using heterogeneous and homogeneous mouse models. Opt. Express 18, 13102-13113. doi: 10.1364/OE.18.013102

Luker, K. E., and Luker, G. D. (2011). Bioluminescence imaging of reporter mice for studies of infection and inflammation. Antiviral Res. 86, 1-17. doi: 10.1016/j.antiviral.2010.02.002

Luwor, R. B., Stylli, S. S., and Kaye, A. H. (2015). Using bioluminescence imaging in glioma research. J. Clin. Neurosci. 22, 779-784. doi: 10.1016/j.jocn.2014.11.001

Ma, R., Taruttis, A., Ntziachristos, V., and Razansky, D. (2009). Multispectral optoacoustic tomography (MSOT) scanner for whole-body small animal imaging. Opt. Express 17, 21414-21426. doi: 10.1364/OE.17.021414

Mahmood, U., Tung, C. H., Bogdanov, A., and Weissleder, R. (1999). Near-infrared optical imaging of protease activity for tumor detection. Radiology 213, 866-870. doi: 10.1148/radiology.213.3.r99dc14866

Martin, A., Aguirre, J., Sarasa-Renedo, A., Tsoukatou, D., Garofalakis, A., Meyer, H., et al. (2008). Imaging changes in lymphoid organs in vivo after brain ischemia with three-dimensional fluorescence molecular tomography in transgenic mice expressing green fluorescent protein in T lymphocytes. Mol. Imaging 7, 157-167.

Masters, B. R. (2009). Encyclopedia of life sciences. West Sussex: John Wiley \& Sons, 1-9. doi: 10.1002/9780470015902.a0022548

McCann, C. M., Waterman, P., Figueiredo, J. L., Aikawa, E., Weissleder, R., and Chen, J. W. (2009). Combined magnetic resonance and fluorescence imaging of the living mouse brain reveals glioma response to chemotherapy. Neuroimage 45, 360-369. doi: 10.1016/j.neuroimage.2008.12.022

Mezzanotte, L., Que, I., Kaijzel, E., Branchini, B., Roda, A., and Löwik, C. (2011). Sensitive dual color in vivo bioluminescence imaging using a new red codon optimized firefly luciferase and a green click beetle luciferase. PLoS ONE 6:e19277. doi: 10.1371/journal.pone.0019277

Mofford, D. M., Reddy, G. R., and Miller, S. C. (2014). Aminoluciferins extend firefly luciferase bioluminescence into the near-infrared and can be preferred substrates over D-luciferin. J. Am. Chem. Soc. 136, 13277-13282. doi: $10.1021 / \mathrm{ja} 505795 \mathrm{~s}$

Montet, X., Figueiredo, J., Alencar, H., Ntziachristos, V., Mahmood, U., and Weissleder, R. (2007). Tomographic fluorescence imaging of tumor vascular volume in mice. Radiology 242, 751-758. doi: 10.1148/radiol.2423052065

Ntziachristos, V., Leroy-Willig, A., and Tavitian, B. (eds). (2007). Textbook of In-Vivo Imaging in Vertebrates. West Sussex: John Wiley \& Sons.

Ntziachristos, V., Ripoll, J., Wang, L. V., and Weissleder, R. (2005). Looking and listening to light: the evolution of whole-body photonic imaging. Nat. Biotechnol. 23, 313-320. doi: 10.1038/nbt1074

Ntziachristos, V., Schellenberger, E. A., Ripoll, J., Yessayan, D., Graves, E., Bogdanov, A., et al. (2004). Visualization of antitumor treatment by means of fluorescence molecular tomography with an annexin V-Cy5.5 conjugate. Proc. Natl. Acad. Sci. U.S.A. 101, 12294-12299. doi: 10.1073/pnas.0401137101

Ntziachristos, V., Tung, C.-H., Bremer, C., and Weissleder, R. (2002). Fluorescence molecular tomography resolves protease activity in vivo. Nat. Med. 8, 757-760. doi: $10.1038 / \mathrm{nm} 729$

Ntziachristos, V., Yodh, A. G., Schnall, M., and Chance, B. (2000). Concurrent MRI and diffuse optical tomography of breast after indocyanine green enhancement. Proc. Natl. Acad. Sci. U.S.A. 97, 2767-2772. doi: 10.1073/pnas.040570597

Razansky, D., Buehler, A., and Ntziachristos, V. (2011). Volumetric real-time multispectral optoacoustic tomography of biomarkers. Nat. Protoc. 6, 1121-9. doi: 10.1038/nprot.2011.351

Razansky, D., Deliolanis, N. C., Vinegoni, C., and Ntziachristos, V. (2012). Deep tissue optical and optoacoustic molecular imaging technologies for pre-clinical research and drug discovery. Curr. Pharm. Biotechnol. 13, 504-522. doi: $10.2174 / 138920112799436258$ 
Ripoll, J. (2012). Principles of Diffuse Light Propagation. Singapore: World Scientific. Sadikot, R. T., and Blackwell, T. S. (2005). Bioluminescence imaging. Proc. Am. Thorac Soc. 2, 537-540, 511-512. doi: 10.1513/pats.200507-067DS

Sauer, M., Hofkens, J., and Enderlein, J. (eds). (2011). Handbook of Fluorescence Spectroscopy and Imaging: From Ensemble to Single Molecules. Weinheim: Wiley$\mathrm{VCH}$

Shcherbo, D., Merzlyak, E. M., Chepurnykh, T. V., Fradkov, A. F., Ermakova, G. V., Solovieva, E. A., et al. (2007). Bright far-red fluorescent protein for whole-body imaging. Nat. Methods 4, 741-746. doi: 10.1038/nmeth1083

Shu, X., Royant, A., Lin, M. Z., Aguilera, T. A., Lev-ram, V., Steinbach, A., et al. (2009). Mammalian expression of infrared fluorescent proteins engineered from a bacterial phytochrome. Science 324, 804-807. doi: 10.1126/science.1168683

Stepanyuk, G. a., Unch, J., Malikova, N. P., Markova, S. V., Lee, J., and Vysotski, E. S. (2010). Coelenterazine-v ligated to $\mathrm{Ca}^{2+}$-triggered coelenterazine-binding protein is a stable and efficient substrate of the red-shifted mutant of Renilla muelleri luciferase. Anal. Bioanal. Chem. 398, 1809-1817. doi: 10.1007/s00216010-4106-9

Stuker, F., Baltes, C., Dikaiou, K., Vats, D., Carrara, L., Charbon, E., et al. (2011a). Hybrid small animal imaging system combining magnetic resonance imaging with fluorescence tomography using single photon avalanche diode detectors. IEEE Trans. Med. Imaging 30, 1265-1273. doi: 10.1109/TMI.2011.2112669

Stuker, F., Ripoll, J., and Rudin, M. (2011b). Fluorescence molecular tomography: principles and potential for pharmaceutical research. Pharmaceutics 3, 229-274. doi: 10.3390/pharmaceutics3020229

Taruttis, A., Morscher, S., Burton, N. C., Razansky, D., and Ntziachristos, V. (2012). Fast multispectral optoacoustic tomography (MSOT) for dynamic imaging of pharmacokinetics and biodistribution in multiple organs. PLOS ONE 7:e30491. doi: 10.1371/journal.pone.0030491

Thomas, N., Li, P., Fleming, B. C., Chen, Q., Wei, X., Pan, X., et al. (2015). Attenuation of cartilage pathogenesis in post-traumatic osteoarthritis (PTOA) in mice by blocking the stromal derived factor 1 receptor (CXCR4) with the specific inhibitor, AMD3100. J. Orthop. Res. 33, 1071-1078. doi: 10.1002/jor.22862

Tran, M. T. N., Tanaka, J., Hamada, M., Sugiyama, Y., Sakaguchi, S., Nakamura, M., et al. (2014). In vivo image analysis using iRFP transgenic mice. Exp. Anim. 63, 311-9. doi: 10.1538/expanim.63.311

Tzoumas, S., Deliolanis, N., Morscher, S., and Ntziachristos, V. (2014). Unmixing molecular agents from absorbing tissue in multispectral optoacoustic tomography. IEEE Trans. Med. Imaging 33, 48-60. doi: 10.1109/TMI.2013.2279994

Vonnemann, J., Beziere, N., Böttcher, C., Riese, S. B., Kuehne, C., Dernedde, J., et al. (2014). Polyglycerolsulfate functionalized gold nanorods as optoacoustic signal nanoamplifiers for in vivo bioimaging of rheumatoid arthritis. Theranostics 4, 629-641. doi: 10.7150/thno.8518

Wang, X., Pang, Y., Ku, G., Xie, X., Stoica, G., and Wang, L. V. (2003). Noninvasive laser-induced photoacoustic tomography for structural and functional in vivo imaging of the brain. Nat. Biotechnol. 21, 803-806. doi: 10.1038/nbt839
Wang, Y., Akiyama, H., Terakado, K., and Nakatsu, T. (2013). Impact of sitedirected mutant luciferase on quantitative green and orange/red emission intensities in firefly bioluminescence. Sci. Rep. 3, 2490. doi: 10.1038/ srep02490

Weissleder, R., Tung, C. H., Mahmood, U., and Bogdanov, A. (1999). In vivo imaging of tumors with protease-activated near-infrared fluorescent probes. Nat. Biotechnol. 17, 375-378. doi: 10.1038/7933

$\mathrm{Xu}, \mathrm{H}$., and Rice, B. W. (2009). In-vivo fluorescence imaging with a multivariate curve resolution spectral unmixing technique. J. Biomed. Opt. 14, 064011. doi: $10.1117 / 1.3258838$

Zhang, G., Liu, F., Pu, H., He, W., Luo, J., and Bai, J. (2014). A direct method with structural priors for imaging pharmacokinetic parameters in dynamic fluorescence molecular tomography. IEEE Trans. Biomed. Eng. 61, 986-990. doi: 10.1109/TBME.2013.2292714

Zhang, G., Liu, F., Zhang, B., He, Y., Luo, J., and Bai, J. (2013a). Imaging of pharmacokinetic rates of indocyanine green in mouse liver with a hybrid fluorescence molecular tomography/x-ray computed tomography system. J. Biomed. Opt. 18, 040505. doi: 10.1117/1.JBO.18.4.040505

Zhang, R., Davis, S. C., Demers, J.-L. H., Glaser, A. K., Gladstone, D. J., Esipova, T. V., et al. (2013b). Oxygen tomography by Čerenkov-excited phosphorescence during external beam irradiation. J. Biomed. Opt. 18, 50503. doi: 10.1117/1.JBO.18.5.050503

Zhang, R., D'Souza, A. V., Gunn, J. R., Esipova, T. V., Vinogradov, S. A., Glaser, A. K., et al. (2015a). Cherenkov-excited luminescence scanned imaging. Opt. Lett. 40, 827-830. doi: 10.1364/OL.40.000827

Zhang, X., Tian, Y., Zhang, C., Tian, X., Ross, A. W., Moir, R. D., et al. (2015b). Nearinfrared fluorescence molecular imaging of amyloid beta species and monitoring therapy in animal models of Alzheimer's disease. Proc. Natl. Acad. Sci. U.S.A. 112, 9734-9739. doi: 10.1073/pnas.1505420112

Zhang, R., Glaser, A., Esipova, T. V, Kanick, S. C., Davis, S. C., Vinogradov, S., et al. (2012). Čerenkov radiation emission and excited luminescence (CREL) sensitivity during external beam radiation therapy: Monte Carlo and tissue oxygenation phantom studies. Biomed. Opt. Express 3, 2381-2394. doi: 10.1364/BOE.3.002381

Conflict of Interest Statement: The authors declare that the research was conducted in the absence of any commercial or financial relationships that could be construed as a potential conflict of interest.

Copyright (C) 2015 Arranz and Ripoll. This is an open-access article distributed under the terms of the Creative Commons Attribution License (CC BY). The use, distribution or reproduction in other forums is permitted, provided the original author(s) or licensor are credited and that the original publication in this journal is cited, in accordance with accepted academic practice. No use, distribution or reproduction is permitted which does not comply with these terms. 